\title{
Karyotype and spermatogenesis in Triatoma lenti (Hemiptera: Triatominae), a potential Chagas vector
}

\author{
K.C.C. Alevi ${ }^{1}$, P.P. Mendonça ${ }^{1}$, M. Succi ${ }^{1}$, N.P. Pereira ${ }^{1}$, J.A. Rosa $^{2}$ and \\ M.T.V. Azeredo-Oliveira ${ }^{1}$
}

${ }^{1}$ Laboratório de Biologia Celular, Departamento de Biologia, Instituto de Biociências, Letras e Ciências Exatas, Universidade Estadual Paulista "Júlio de Mesquita Filho", São José do Rio Preto, SP, Brasil ${ }^{2}$ Laboratório de Parasitologia, Departamento de Ciências Biológicas, Faculdade de Ciências Farmacêuticas, Universidade Estadual Paulista "Júlio de Mesquita Filho", Araraquara, SP, Brasil

Corresponding author: K.C.C. Alevi

E-mail: kaiochaboli@hotmail.com

Genet. Mol. Res. 11 (4): 4278-4284 (2012)

Received March 29, 2012

Accepted August 6, 2012

Published December 17, 2012

DOI http://dx.doi.org/10.4238/2012.December.17.3

\begin{abstract}
All species of Triatominae are susceptible to infection by Trypanosoma cruzi (Kinetoplastida: Trypanosomatidae) and consequently, potential insect vectors of Chagas disease. Currently, there are 140 known species of triatomine bugs, which can be grouped into specific species complexes. The species Triatoma lenti (Hemiptera: Triatominae) is found only in Brazil and is considered a potential vector of Chagas disease. We karyotyped male T. lenti and examined its spermatogenesis in detail. The karyotype was found to be $2 \mathrm{n}=20 \mathrm{~A}$ $+\mathrm{XY}$, demonstrating that this organism has the modal chromosome set found in triatomines. This new information concerning males
\end{abstract}


of this species contributed to biological data that will be useful for understanding this potentially important Chagas disease vector.

Key words: Triatomine; Cytogenetics; Holocentric chromosomes; Meiosis; Brasiliensis subcomplex

\section{INTRODUCTION}

All species of Triatominae are susceptible to infection by Trypanosoma cruzi and consequently, are potential insect vectors of Chagas disease. Infection occurs from feeding on mammalian infected blood, with all instars capable of parasite ingestion since blood meal is required at all stages of life in triatomines (Noireau et al., 2009). Currently, there are 140 known species of Triatominae, which is divided into 5 tribes and distributed across 15 genera (Schofield and Galvão, 2009), with the genera Triatoma, Panstrongylus, and Rhodnius being epidemiologically the most important.

Triatominae species can be grouped into specific complexes according to morphological similarities, geographical distribution, possibility of hybridization, and molecular proximity. Because of several possible approaches on the basis of the parameters evaluated, up to now there is no consensus about the standardization of the group into complexes and subcomplexes (Schofield and Galvão, 2009).

The systematic and specific identification of the insects has been based primarily on morphological observations, mainly with light microscopy (Lent and Wygodzinsky, 1979). However, analysis of morphological parameters alone cannot always answer all taxonomic questions, as in the case of species such as the Brasiliensis subcomplex and Rhodnius genus (Monteiro et al., 2001; Jurberg, 2003). In order to resolve these issues, more sensitive cytogenetic approaches are needed to distinguish among these species.

In cytogenetics, biological models of triatomine bugs are important because they have holocentric chromosomes, which have diffuse kinetochores distributed along the chromosomes and not a single kinetochore located in the centromeric region. These insects also perform an unusual meiosis in which the segregation of sex chromosomes is post-reductional (Barth, 1956; Ueshima, 1966).

Most of the cytogenetic results for triatomines were obtained on the basis of chromosome comparisons that relied on conventional staining. In these studies, the comparison criteria were often the number, morphology, and disposition of chromosomes in the metaphase plate. The data obtained from these investigations provided the first indications of the processes that occurred during chromosomal evolution in these insects (Pérez et al., 1992; Panzera et al., 2010).

According to Monteiro et al. (2001), it is difficult to explain the Brasiliensis subcomplex classification by using morphological parameters alone, because these species are cryptic. Thus, cytogenetic studies can be considered an important tool for the differentiation of these species (Pérez et al., 1992).

The species Triatoma lenti, described by Sherlock and Serafim in 1967, is a Hemiptera that belongs to the Infestans group, Infestans complex, and Brasiliensis subcomplex (Schofield and Galvão, 2009). This subcomplex is present in South America and consists of 9 species: T. brasiliensis, T. juazeirensis, T. melanica, T. melanocephala, T. petrochiae, T. lenti, T. sherlocki, T. tibiamaculata, and T. vitticeps (Schofield and Galvão, 2009). 
T. lenti is found only in Brazil and is endemic to Bahia and Goiás (Carcavallo et al., 1999), and according to Sherlock and Guitton (1974), it was found infected with the protozoan T. cruzi.

To elucidate the mechanisms involved in the cytogenetic characteristic of these insects during cell division and to understand and differentiate the organisms of the Brasiliensis subcomplex, we have described for the first time the karyotype of $T$. lenti and examined in detail its spermatogenesis. These data will aid in the evolutionary study of the insects, and thus, help us understand this important insect vector of Chagas disease and contribute to the control of the populations.

\section{MATERIAL AND METHODS}

The species analyzed was $T$. lenti. For the study, 15 specimens were used. The insects were provided by Triatominae Insectarium of São Paulo State University (UNESP, city of Araraquara, Brazil). The seminiferous tubules of the adult males underwent lacto-acetic orcein staining (De Vaio et al., 1985, with modifications). The slides were analyzed using a light microscope (Jenaval-Zeiss), which was coupled to a digital camera and an AxioVision LE 4.8 image analyzer (Copyright ${ }^{\mathcal{O}}$ 2006-2009 Carl Zeiss Imaging Solutions Gmb H). The images were magnified by $1000 \mathrm{X}$.

\section{RESULTS}

Through the cytogenetic technique of lacto-acetic orcein staining, it was possible to determine the diploid chromosome set of T. lenti. On the basis of the meiotic metaphases, the studied species showed the karyotype $2 n=22(20 \mathrm{~A}+\mathrm{XY})$, i.e., 10 pairs of autosomes and 2 sex chromosomes in metaphase I and 10 autosomes and 1 pair of sex chromosomes in metaphase II (Figure 1). Karyotype analysis showed that some autosomes were larger than the others. The sex chromosomes were the smallest in the chromosome set and differed in size and coloration, being the largest and most heteropycnotic. The polyploid nuclei of the nutritive cells of the seminiferous tubules showed a higher degree of heteropycnosis and a more evident corpuscle (Figure 2A). In relation to the spermatogenesis of T. lenti, the following was observed. During meiotic division, and more specifically during the diffuse stage of prophase I, it was possible to monitor chromatin condensation. In the initial diffuse stage (Figure 2B and $\mathrm{C}$ ), the condensed chromatin presented some regions as more heteropycnotic than others. An individualization of chromosomes was noted in nuclei in the intermediate diffuse stage (Figure 2D) and final diffuse stage (Figure 2E). In metaphase I, 2 univalent sex chromosomes were located in the center of the ring formed by autosomal bivalents (Figure 2F). In this phase, the condensed chromosomes showed varying degrees of heteropycnosis among them, with some autosomes being more heteropycnotic than the sex chromosomes. In metaphase II, chromosomes showed the same ring disposition (Figure 2G). Different degrees of heteropycnosis were observed on chromosomes during metaphases analyzed in lateral view (Figure $2 \mathrm{H}$ ). In the meiotic metaphase, the difference in size among the heterochromosomes was evident. Cells in the telophase did not show the late migration phenomenon (Figure 2I). 


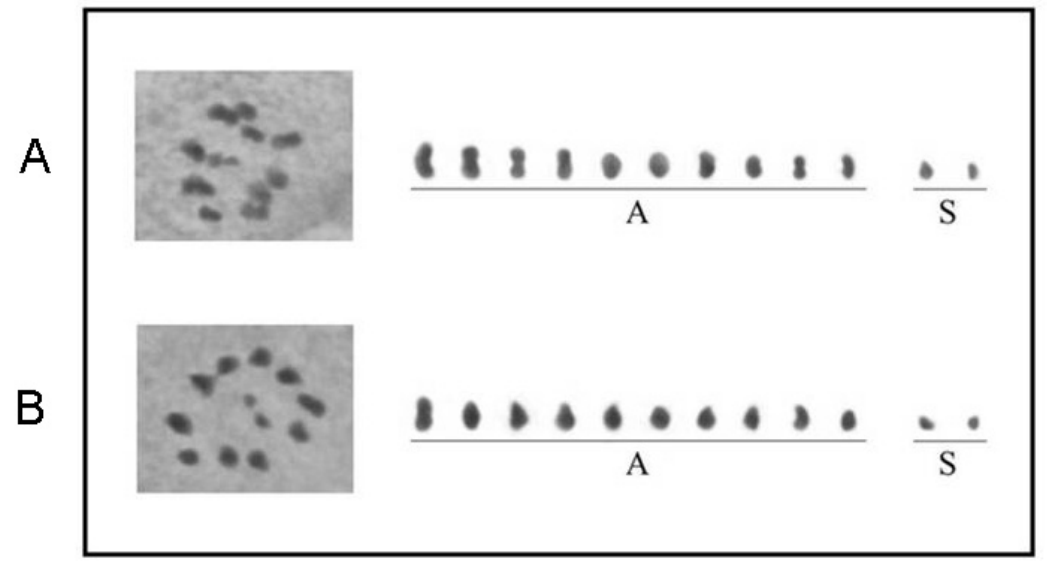

Figure 1. Karyotype of Triatoma lenti. A. Circular metaphase of first meiotic division and respective karyotype with 10 bivalent autosomes and 2 sex chromosomes (XY). B. Circular metaphase of second meiotic division and respective karyotype $\mathrm{n}=(10 \mathrm{~A}+\mathrm{XY})$. $\mathrm{A}=$ autosomes; $\mathrm{S}=$ sex chromosomes.

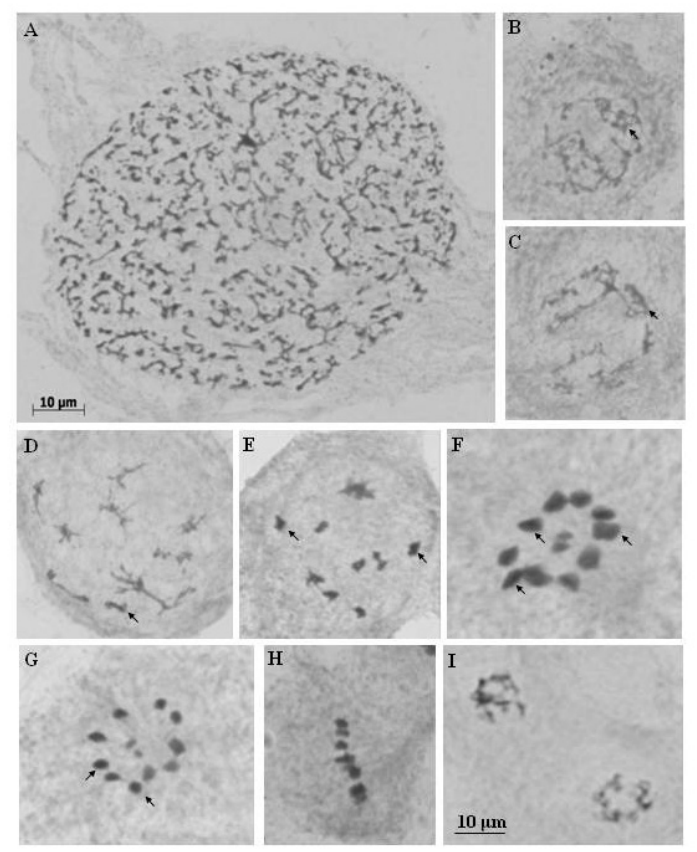

Figure 2. Seminiferous tubule of Triatoma lenti stained by lacto-acetic orcein. A. Polyploidy nuclei with a high degree of heteropycnose and an evident corpuscle. B. C. Diffuse stages. Note the heteropycnotic regions (arrows). D. Intermediate diffuse stage (arrow). E. Final diffuse stage with the chromosomes more condensed, showing different degrees of heteropycnose (arrows). F. Metaphase I with 10 bivalent autosomes and 2 sex chromosomes. Some autosomes were more heteropycnotic (arrows). G. Metaphase II in ring configuration (arrow). H. Metaphases in lateral view. I. Telophase. 


\section{DISCUSSION}

Cytogenetic studies have significantly contributed to our understanding of the taxonomy and systematics of insects, despite the small size of the chromosomes and their holocentric nature, which hinder in the detection of their rearrangement or changes in the structures (Camargo et al., 2006).

Cytogenetic analysis of $T$. lenti permitted us to observe that these organisms have holocentric chromosomes, supporting the hypothesis that all Heteroptera have holocentric chromosomes (Souza and Itoyama, 2006). The Heteroptera holocentric chromosomes are an excellent material for study and are used as models in investigations, since they have peculiar and interesting characteristics such as kinetic activity restricted to the chromosome ends, which is presumed to occur during chiasma finalization. These insects also undergo inverted meiosis for sex chromosomes, since the first division is reductional for autosomes and equational for the sex chromosomes, while the second division is equational for autosomes and reductional for sex chromosomes (Ueshima, 1979).

Through this technique, it was possible to identify the condensed chromatin, since this conventional cytogenetic technique enables a refined differentiation between euchromatin and heterochromatin. This is because of the stronger staining of euchromatin than heterochromatin, since heterochromatin has higher compression and more proteins than euchromatin (Azeredo-Oliveira, 1990).

The basic number of chromosomes for triatomines is $2 \mathrm{n}=22$, with 10 pairs of autosomes and 1 pair of sex chromosomes. However, there are species with multiple sex chromosomes (XY, $\left.\mathrm{X}_{1} \mathrm{X}_{2} \mathrm{Y}, \mathrm{X}_{1} \mathrm{X}_{2} \mathrm{X}_{3} \mathrm{Y}\right)$, caused by fragmentation of the original $\mathrm{X}$ (Ueshima, 1966).

The analysis of metaphases in frontal view allows the description of the karyotype and analysis of chromosomes in Heteroptera, for in this phase of cell division, chromosomes are highly compressed, meaning that they are in their highest degree of condensation. Thus, on the basis of the metaphases analyzed, we observed that males of $T$. lenti have the karyotype $2 n=22(20 A+X Y)$, demonstrating thereby that this organism has the modal chromosome set found in triatomines. It is believed that $60 \%$ of the species analyzed of triatomine bugs have a diploid number of chromosomes: $2 \mathrm{n}=20 \mathrm{~A}+\mathrm{XY}$ (Tavares and Azeredo-Oliveira, 1997a).

Among the triatomines, there is little variation in the size of the chromosomes (De Vaio et al., 1985). However, analysis of the karyotype of $T$. lenti demonstrates that the chromosomes show variation in size and degree of heteropycnosis. De Vaio et al. (1985) performed cytogenetic analysis of 3 species of insects and noted that these organisms had 1 to 3 larger and more heteropycnotic chromosomes.

The Brasiliensis subcomplex is composed of 9 species (Schofield and Galvão, 2009), and the karyotypes of 7 species have already been described: T. brasiliensis, T. juazeirensis, T. melanica, T. petrochiae, T. tibiamaculata, T. vitticeps (Panzera et al., 2010), and T. melanocephala (Alevi et al., 2012). Here, on the basis of the analysis of the meiotic metaphase, the karyotype of $T$. lenti has been described for the first time. This organism, like T. brasiliensis, $T$. juazeirensis, T. melanica, and T. petrochiae, has a diploid chromosome set that consists of $2 \mathrm{n}=$ 22 chromosomes $(2 n=20 \mathrm{~A}+\mathrm{XY})$, demonstrating that $T$. lenti, with respect to karyotype analysis, is close to most species that have already been analyzed cytogenetically in this subcomplex.

The species T. tibiamaculata and T. vitticeps share some morphological similarities with the species of the Brasiliensis subcomplex, but when analyzed cytogenetically and on a 
molecular basis, they are related to a greater extent to the Triatominae of North America, such as T. tibiamaculata and T. vitticeps, which have a system of multiple sex determination because of multiple sex chromosomes. In other words, the karyotype of male T. tibiamaculata is $2 \mathrm{n}$ $=20 \mathrm{~A}+\mathrm{X}_{1} \mathrm{X}_{2} \mathrm{Y}$ (Panzera et al., 1996) and the karyotype T. vitticeps is $2 \mathrm{n}=20 \mathrm{~A}+\mathrm{X}_{1} \mathrm{X}_{2} \mathrm{X}_{3} \mathrm{Y}$ (Schreiber and Pellegrino, 1950). Mitochondrial DNA sequences also confirm the close phylogenetic relationship between these Hemiptera and the ones present in North America (Schofield and Galvão, 2009). Alevi et al. (2012) recently described the karyotype of T. melanocephala $\left(2 \mathrm{n}=20 \mathrm{~A}+\mathrm{X}_{1} \mathrm{X}_{2} \mathrm{X}_{3} \mathrm{Y}\right)$ and suggested the exclusion of T. melanocephala, T. vitticeps and $T$. tibiamaculata from the subcomplex by similarity with the triatomines in North America.

Analysis of the seminiferous tubule cells of T. lenti, allowed the observation of chromosome characteristics and the meiotic behavior of this insect. The polyploid nuclei showed a high degree of heteropycnosis and a more evident corpuscle. Other species of Heteroptera analyzed cytogenetically also demonstrated these characteristics in the polyploid nucleus (Tavares and Azeredo-Oliveira, 1997b).

The meiotic process of holocentric chromosomes was corroborated by the analysis of the spermatogenesis of T. lenti. The chromosomes showed normal patterns found in triatomines, such as the arrangement of sex chromosomes in the center of the ring formed by autosomes (De Vaio et al., 1985; Pérez et al., 1992). The inverted meiosis phenomenon for heterochromosomes was also confirmed in the present study.

This study contributed to knowledge on Triatominae. These data allow the Brasiliensis subcomplex to be understood better, since it is composed of cryptic species. Therefore, accumulation of more biological data will make it easier to understand the evolution and biology of these insects, which are important insect vectors of Chagas disease.

\section{ACKNOWLEDGMENTS}

Research supported by Fundação de Amparo à Pesquisa do Estado de São Paulo (FAPESP), Conselho Nacional de Desenvolvimento Científico e Tecnológico (CNPq), Coordenação de Aperfeiçoamento Pessoal de Nível Superior (CAPES) and Fundação para o Desenvolvimento da UNESP (FUNDUNESP).

\section{REFERENCES}

Alevi KC, Mendonca PP, Pereira NP, Rosa JA, et al. (2012). Karyotype of Triatoma melanocephala Neiva and Pinto (1923). Does this species fit in the Brasiliensis subcomplex? Infect. Genet. Evol. 12: 1652-1653.

Azeredo-Oliveira MTV (1990). Estudo Citogenético em Túbulos de Malpighi, Glândulas Salivares e Testículos de Triatomíneos (Triatominae, Heteroptera). Master's thesis, IBILCE/UNESP, São José do Rio Preto.

Barth R (1956). Estudos anatômicos e histológicos sôbre a subfamília Triatominae (Heteroptera, Reduviidae). VI parte: Estudo comparativo sôbre a espermiocitogênese das espécies mais importantes. Mem. Inst. Oswaldo Cruz 54: 599-616.

Camargo M, Duque-Correa MA and Berrio A (2006). A micro-spreading improvement for spermatogenic chromosomes from Triatominae (Hemiptera-Reduviidae). Mem. Inst. Oswaldo Cruz 101: 339-340.

Carcavallo RU, Curto de Casas SI, Sherlock IA and Galíndez-Girón I (1999). Geographical Distribution and AltiLatitudinal Dispersion. In: Atlas of Chagas Disease Vectors in the America (Carcavallo RU, Galíndez-Girón I, Jurberg J and Lent H, eds.). Editora Fiocruz, Rio de Janeiro, 747-792.

De Vaio ES, Grucci B, Castagnino AM and Franca ME (1985). Meiotic differences between three triatomine species (Hemiptera: Reduviidae). Genetica 67: 185-191.

Juberg J (2003). Ferramentas usadas em taxonomia de Triatomíneos o uso múltiplo. Entomol. Vectores 10: 497-509.

Lent H and Wygodzinsky P (1979). Revision of the Triatominae (Hemiptera: Reduviidae) and their significance as vector 
of Chagas's disease. Bull. Am. Mus. Nat. Hist. 163: 123-520.

Monteiro FA, Escalante AA and Beard CB (2001). Molecular tools and triatomine systematics: a public health perspective. Trends Parasitol. 17: 344-347.

Noireau F, Diosque P and Jansen AM (2009). Trypanosoma cruzi: adaptation to its vectors and its hosts. Vet. Res. 40: 26.

Panzera F, Perez R, Hornos S, Panzera Y, et al. (1996). Chromosome numbers in the Triatominae (Hemiptera-Reduviidae): a review. Mem. Inst. Oswaldo Cruz 91: 515-518.

Panzera F, Perez R, Panzera Y, Ferrandis I, et al. (2010). Cytogenetics and genome evolution in the subfamily Triatominae (Hemiptera, Reduviidae). Cytogenet. Genome Res. 128: 77-87.

Pérez R, Panzera Y, Scafiezzo S, Mazzella MC, et al. (1992). Cytogenetics as a tool for triatomine species distinction (Hemiptera-Reduviidae). Mem. Inst. Oswaldo Cruz 87: 353-361.

Schofield CJ and Galvão C (2009). Classification, evolution, and species groups within the Triatominae. Acta Trop. 110 88-100.

Schreiber G and Pellegrino J (1950). Eteropicnosi di autosomi come possible meccanismo di speciazione. Sci. Genet. 3 : 215-226.

Sherlock IA and Guitton N (1974). Fauna Triatominae do Estado da Bahia Brasil III - notas sobre ecótopos silvestres e o gênero Psammolestes. Mem. Inst. Oswaldo Cruz 72: 91-101.

Souza HV and Itoyama MM (2006). Diferentes sistemas cromossômicos de determinação do sexo em Heteroptera. Rev. UNORP 13: 80-87.

Tavares MG and Azeredo-Oliveira MTV (1997a). Cytogenetics studies on holocentric chromosomes of five species of triatomines (Heteroptera: Reduviidae). Cytobios 89: 51-61.

Tavares MG and Azeredo-Oliveira MTV (1997b). Pattern of nucleolar activity during spermatogenesis in triatomines (Heteroptera, Reduviidae) as analyzed by silver staining. Cytobios 89: 93-103.

Ueshima N (1966). Cytotaxonomy of the Triatominae (Reduviidae: Hemiptera). Chromosoma 18: 97-122.

Ueshima N (1979). Hemiptera II: Heteroptera. In: Animal Cytogenetics (John B, ed.). Gebrüder Borntraeger, Berlim, $1-117$. 